\title{
Tumor cell behaviour modulation by mesenchymal stromal cells
}

\author{
Lucia Kucerova*, Miroslava Matuskova, Kristina Hlubinova, Veronika Altanerova and Cestmir Altaner
}

\begin{abstract}
Background: Human mesenchymal stromal cells (MSC) hold a promise for future cell-based therapies due to their immunomodulatory properties and/or secretory activity. Nevertheless non-neoplastic tumor compartment could also originate from MSC. We aimed to show whether multipotent MSC derived from human adipose tissue (AT-MSC) could create tumor cell-protective milieu and affect tumor cell behaviour in vitro and in vivo.

Results: Here we have demonstrated tumor-promoting effect of AT-MSC on human melanoma A375 cells. AT-MSC coinjection mediated abrogation of tumor latency and supported subcutaneous xenotransplant growth from very low melanoma cell doses. Tumor incidence was also significantly increased by AT-MSC-derived soluble factors. AT-MSC supported proliferation, suppressed apoptosis and modulated melanoma cell responses to cytotoxic drugs in vitro. Expression and multiplex cytokine assays confirmed synergistic increase in VEGF that contributed to the AT-MSCmediated support of A375 xenotransplant growth. Production of G-CSF and other factors implicated in formation of supportive proinflammatory tumor cell microenvironment was also confirmed. SDF-1a/CXCR4 signalling contributed to tumor-promoting effect of systemic AT-MSC administration on A375 xenotransplants. However, no support was observed for human glioblastoma cells 8MGBA co-injected along with AT-MSC that did not sustain tumor xenotransplant growth in vivo. Tumor-inhibiting response could be attributed to the synergistic action of multiple cytokines produced by AT-MSC on glioblastoma cells.

Conclusions: Herein we provide experimental evidence for MSC-mediated protective effect on melanoma A375 cells under nutrient-limiting and hostile environmental conditions resulting from mutual crosstalk between neoplastic and non-malignant cells. This tumor-favouring effect was not observed for the glioblastoma cells 8MGBA. Collectively, our data further strengthen the need for unravelling mechanisms underlying MSC-mediated modulation of tumor behaviour for possible future MSC clinical use in the context of malignant disease.
\end{abstract}

\section{Background}

Mesenchymal stromal cells (MSC) represent a heterogeneous population of multi-potent cells with beneficial properties for regenerative processes and/or immunomodulation [1]. Therapeutic benefit for patients suffering from a wide range of severe pathologic conditions was reported in clinical trials employing MSC and derivatives thereof [2-5]. However, MSC therapy may also bring adverse effects such as increased recurrence rate of hematologic malignancy as recently reported [6].

Increasing evidence has shown that MSC might play a role in the tumor pathogenesis and progression. Tumor

* Correspondence: exonkuce@savba.sk

${ }^{1}$ Laboratory of Molecular Oncology, Cancer Research Institute, Slovak Academy of Sciences, Bratislava, Slovakia

Full list of author information is available at the end of the article behaviour is affected by non-neoplastic compartment of stroma composed from extracellular matrix, blood vessels, connective tissue, MSC, immune and inflammatory cells dynamically interlinked with tumor parenchyma [710]. Its growth results from the neoplastic cells' interaction with the complex stromal compartment and components thereof can be derived from progenitors residing in the bone-marrow $[11,12]$.

Mutual cellular interactions of MSC and tumor cells were investigated in several studies to unravel the MSC effect on tumor properties. Human MSC maintained under standard culture conditions were shown to be nontumorigenic per se, however, several reports presented their capability to modulate tumor microenvironment thus having an impact on the tumor behaviour [13]. MSC produce cytokines with proangiogenic action, MSC can 
give rise to endothelial-like or pericyte-like cells contributing to tumor vasculature formation and stabilization when recruited to the site of tumor formation [12]. MSC exhibited a capability to differentiate into carcinomaassociated fibroblasts upon culture under the influence of tumor-cell produced soluble factors in vitro consequently leading to tumor growth support in vivo [14]. Unmanipulated human MSC were shown to increase the metastatic potential of breast cancer cells rather than significant tumor growth support [15]. Several other studies aimed on modelling of the interplay between tumor cells and non-tumorigenic stromal cells have shown various MSC effects on tumor cell behaviour in vitro and in vivo. MSC strongly inhibited proliferation of malignant cells of hematopoietic origin in vitro, nevertheless significantly increased BV173 tumor incidence in vivo [16]. Authors hypothesized that MSC were capable to preserve selfrenewal potential of leukemic cells by mimicking cancer stem cell niche. Increased tumor incidence rather than change in tumor growth rate was reported for renal cell carcinoma, colon carcinoma and melanoma cells coinjected with MSC in syngeneic model [17]. Moreover, systemically administered MSC increased tumor incidence and allowed for the proliferation of renal carcinoma cells. Zhu et al. have shown similar effects of MSC-favoured tumor growth for two colon carcinoma cell lines upon coinjection with bone marrow-derived human MSC on xenogeneic model [18]. Glioma outgrowth was significantly supported by intracranial or subcutaneous tumor cell coimplantation together with human adipose tissue derived MSC [19].

On the contrary, there were several reports to show the anti-tumor effect of MSC. Khakoo et al. have used systemic MSC injection to inhibit the growth of Kaposi's sarcoma subcutaneous xenotransplant [20]. Prolongation of latent tumor time and tumor size decrease was shown for hepatoma cells coinjected with immortalized human fetal MSC [21]. Furthermore, MSC coimplantation with breast cancer cells resulted in inhibited tumor growth and reduced metastasis in vivo. [22]. Intratumoral injection of rat MSC prolonged survival in 9L glioma-bearing rats as a consequence of retarded tumor growth [23]. Several studies employing MSC as tumor-targeting delivery vehicles including our observations have reported no significant influence on tumor growth in vivo [24-26].

In our present study we aimed to examine the influence of human adipose tissue derived mesenchymal stromal cells (AT-MSC) on tumor development. AT-MSC could protect human melanoma cells from nutrient limitations and/or cytotoxic effects by apoptosis inhibition in vitro. Tumor-favouring effects on melanoma A375 xenografts were highly AT-MSC dose dependent in vivo and tumor incidence increase in immunocompromised host recapitulated data from syngeneic model reported previously
[17]. However, AT-MSC did not increase proliferation of glioblastoma 8MGBA cells and could suppress glioblastoma 8MGBA xenograft growth in vivo. We hypothesized that the diversity in and responsiveness to paracrine factors produced by AT-MSC and given tumor cell lines resulted in differential tumor microenvironment composition affecting outcome of mutual tumor cell/AT-MSC interplay.

\section{Results}

AT-MSC support melanoma growth and increase tumor incidence in vivo

In order to determine, whether AT-MSC exhibit tumor supportive or inhibitory effect on melanoma cells, we first admixed AT-MSC to melanoma cell doses with $100 \%$ tumor penetrance. AT-MSC coimplanted with M4Beu melanoma cells significantly decreased time to $100 \%$ tumor onset in comparison to M4Beu alone. Average tumor burden was higher in M4Beu/AT-MSC (ratio 5:1) group in comparison to the control group (Fig. 1A and 1B). Similarly, A375/AT-MSC (5:1 ratio) injected group of animals also exhibited shortened time to tumor onset from 10 days in A375 alone-group to 3 days concomitantly exhibiting tendency to higher average tumor volume in AT-MSC coinjected groups (Fig. 1C and 1D).

Next, we aimed to determine whether AT-MSC could affect tumor incidence for limited amounts of tumor cells, which leave almost all animals long term tumor free $\left(1 \times 10^{5}\right.$ A375). AT-MSC coinjection significantly increased tumor incidence and tumor growth in groups coinjected with $10: 1$ or 1:1 AT-MSC to A375 cell ratio (Fig. 2A). Moreover, soluble AT-MSC produced factors were sufficient to increase tumor incidence, if AT-MSC conditioned cell-free medium was used for the A375 cell resuspension, which indicated a role of paracrine factors in tumor-promoting action. Tumor volume as a measure of tumor burden within the treatment groups was proportional to the amount of coinjected AT-MSC and significantly higher in comparison to tumor burden in control group due to earlier latency abrogation (Fig. 2BC). Thus we conclude that AT-MSC could abrogate tumor latency for as low as 100,000 melanoma A375 cells, which would not produce tumors if injected alone in immunocompromised host.

\section{AT-MSC can support proliferation, protect melanoma A375 cells from nutrient deprivation or cytotoxic cellular stress in vitro}

Coculture experiments in vitro were designed to characterize the interaction between tumor cells A375 and ATMSC to unravel mechanism responsible for the protumorigenic effect. In order to determine the effect on tumor cell proliferation, A375 cells stably expressing EGFP (EGFP-A375) were mixed with increasing amounts of 

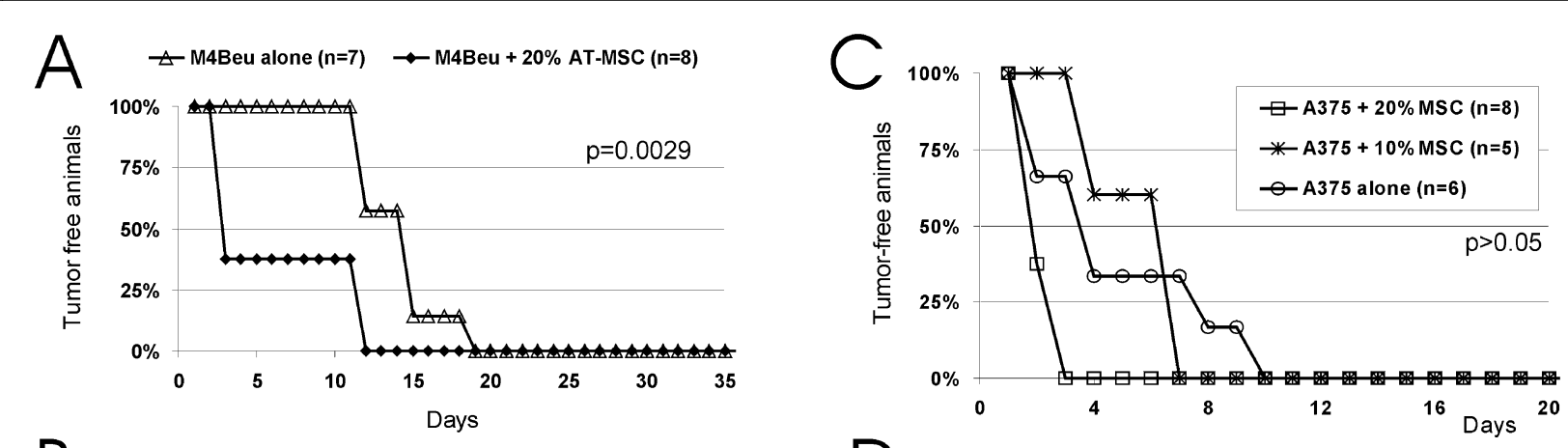

B
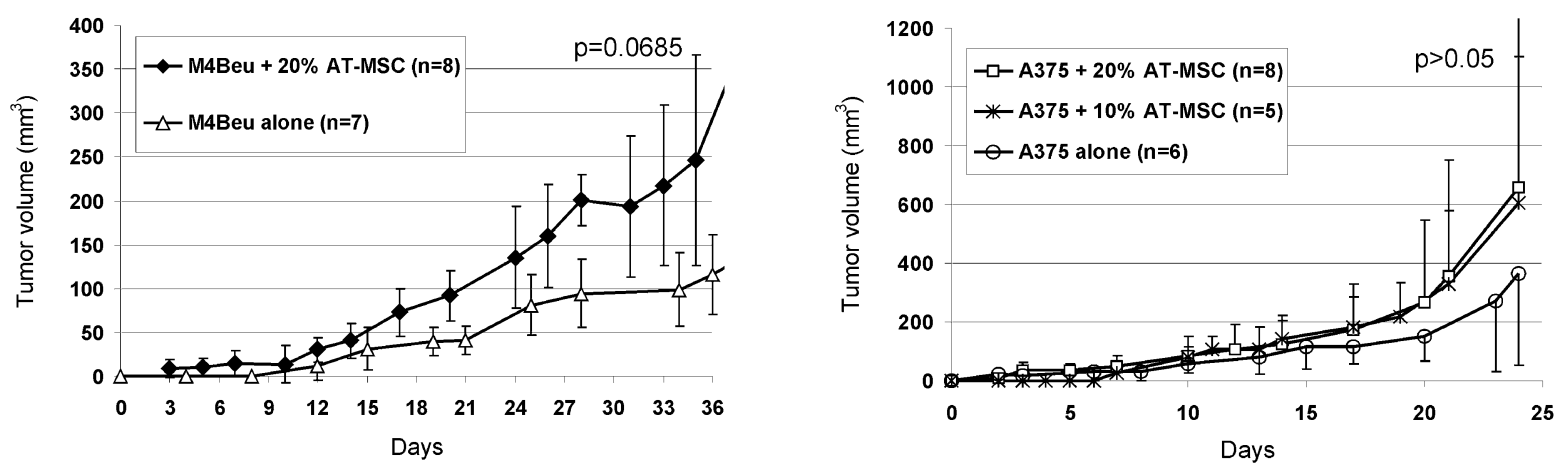

Figure 1 AT-MSC coinjection with high melanoma cell dose changes the time of xenograft onset and growth. A.-B. 20\% AT-MSC co-administered s.c. with $1 \times 10^{6} \mathrm{M} 4 \mathrm{Beu}$ significantly shortened time to $100 \%$ tumor onset from day 19 (control) to day 12 , but did not significantly increase tumor burden. C.-D. $10 \%$ or $20 \%$ AT-MSC admixed with $1.5 \times 10^{6}$ A375 melanoma cells also shortened time of xenograft onset and increased tumor burden in nude mice.

AT-MSC or conditioned medium produced from corresponding amount of AT-MSC cells. Output fluorescence was proportional to the number of EGFP-A375 cells and the amount of admixed AT-MSC cells did not interfere with output fluorescence. Soluble factors supported EGFP-A375 proliferation even in serum-limiting culture conditions although to much lesser extent in comparison to directly cocultured cells (Fig. 3A). AT-MSC also protected tumor cells from serum-deprivation induced apoptosis (A375/AT-MSC ratio 10:1, Fig. 3B). Direct cocultures of melanoma cells with AT-MSC (ratio 10:1) did not exhibit change in effector caspase activation induced by cytotoxic drugs in standard serum concentrations (not shown). However, doxorubicin and cisplatin treatment under serum deprivation conditions resulted in AT-MSC-mediated significant decrease in effector caspase-3/7 activation consequently leading to decrease in proportion of apoptotic and dead cells (Fig. 3C and 3D). Our data suggest that AT-MSC may assist tumor cells to sustain cellular stress such as nutrient deprivation and/or cytotoxicity. Indeed, in the presence of AT-MSC there was a significant increase of A375 colony-forming ability even in the absence of cell-cell contact in vitro (Fig. 4A). No such effect was observed when AT-MSC were added to the cultures three days later, so AT-MSC seemed to initiate colony growth at early stage. Indirect cocultures of melanoma and AT-MSC cells enabled us to analyze the expression of growth factors and receptors that was previously implicated to play a role in AT-MSC/tumor cell interactions. Quantitative analysis unravelled increased CCL5 production from AT-MSC in response to melanoma cells (Fig. 4B). Sustained expression of several potential prosurvival and proangiogenic factors and their cognate receptors in AT-MSC and A375 was demonstrated even upon 3 day coculture (Fig. 4C).

Multiplex cytokine analysis was performed in order to quantitatively evaluate a paracrine signalling in tumor/ AT-MSC cocultures. A wide plethora of cytokines and chemokines was detected to be secreted from both cell types. Combined coculture of these cells exhibited additive or slightly synergistic effects for most of them, nevertheless significantly increased secretion of G-CSF was detected, apparently as a response of melanoma cells, and increased VEGF production proportional to the AT-MSC number in cocultures (Fig. 5A, B). Taken together mutual crosstalk between melanoma and AT-MSC within the tumor microenvironment results in formation of proinflammatory and proangiogenic cellular milieu resulting in 

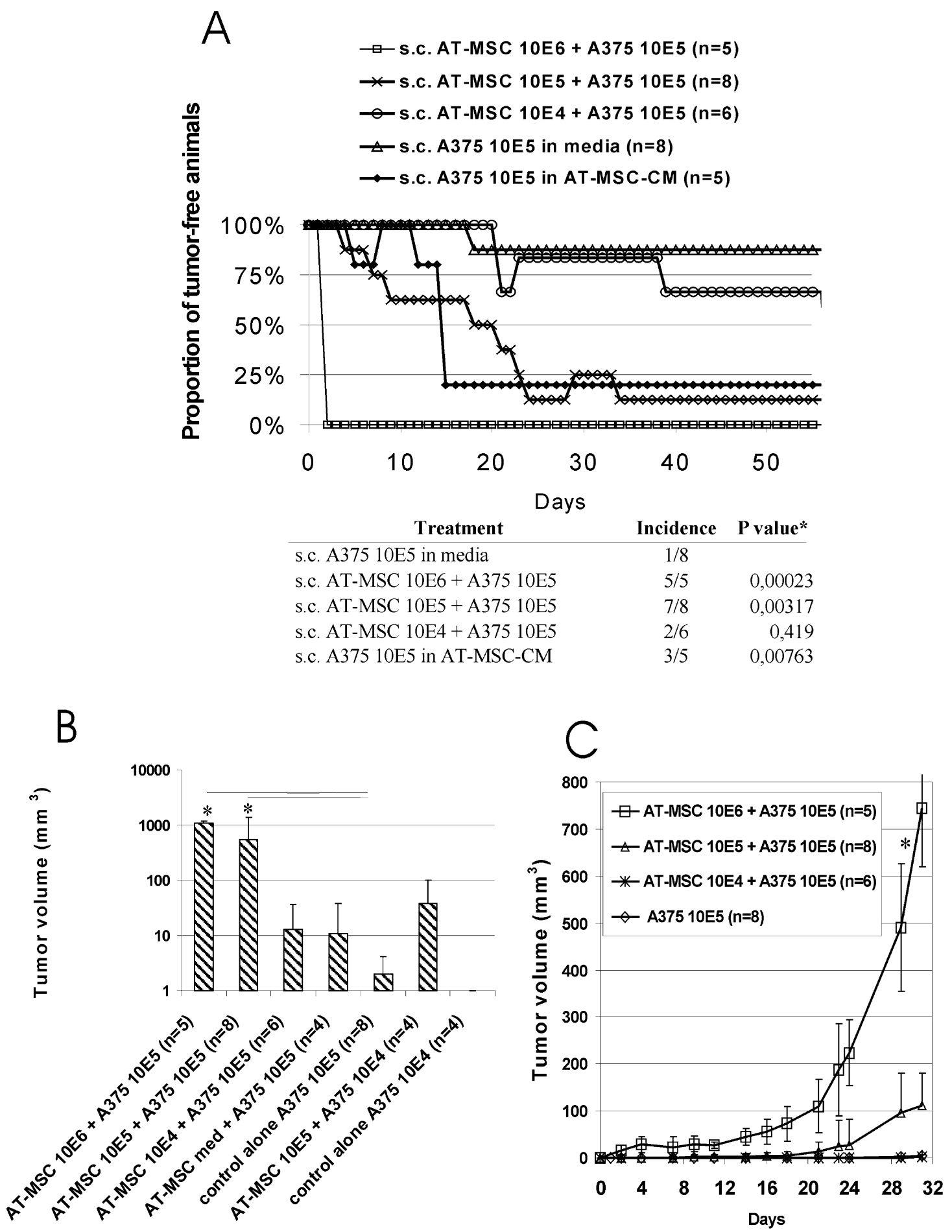

Figure 2 AT-MSC abrogated tumor dormancy of low melanoma cell dose and supported tumor growth. $1 \times 10^{5}$ or $1 \times 10^{4} \mathrm{~A} 375$ cells resuspended in serum-free culture media were injected s.c. either alone or admixed to AT-MSC in ratios 10:1, 1:1 or 1:10. Low A375 cell dose $\left(1 \times 10^{5}\right.$ s.c.) resuspended in cell-and-serum free AT-MSC-conditioned media was injected in a separate groups of animals. A. AT-MSC admixed to the A375 at a ratio 1:1 or 10:1 significantly increased tumor incidence in nude mice. AT-MSC conditioned media also abrogated tumor dormancy of low A375 melanoma dose. B. Tumor burden in 10:1 and 1:1 AT-MSC coinjected groups was significantly higher in comparison to A375 cell-induced xenografts by day 40. C. AT-MSC co-administration resulted in melanoma xenograft tumor growth support proportional to the AT-MSC cell dose (*P $<0.05)$. 


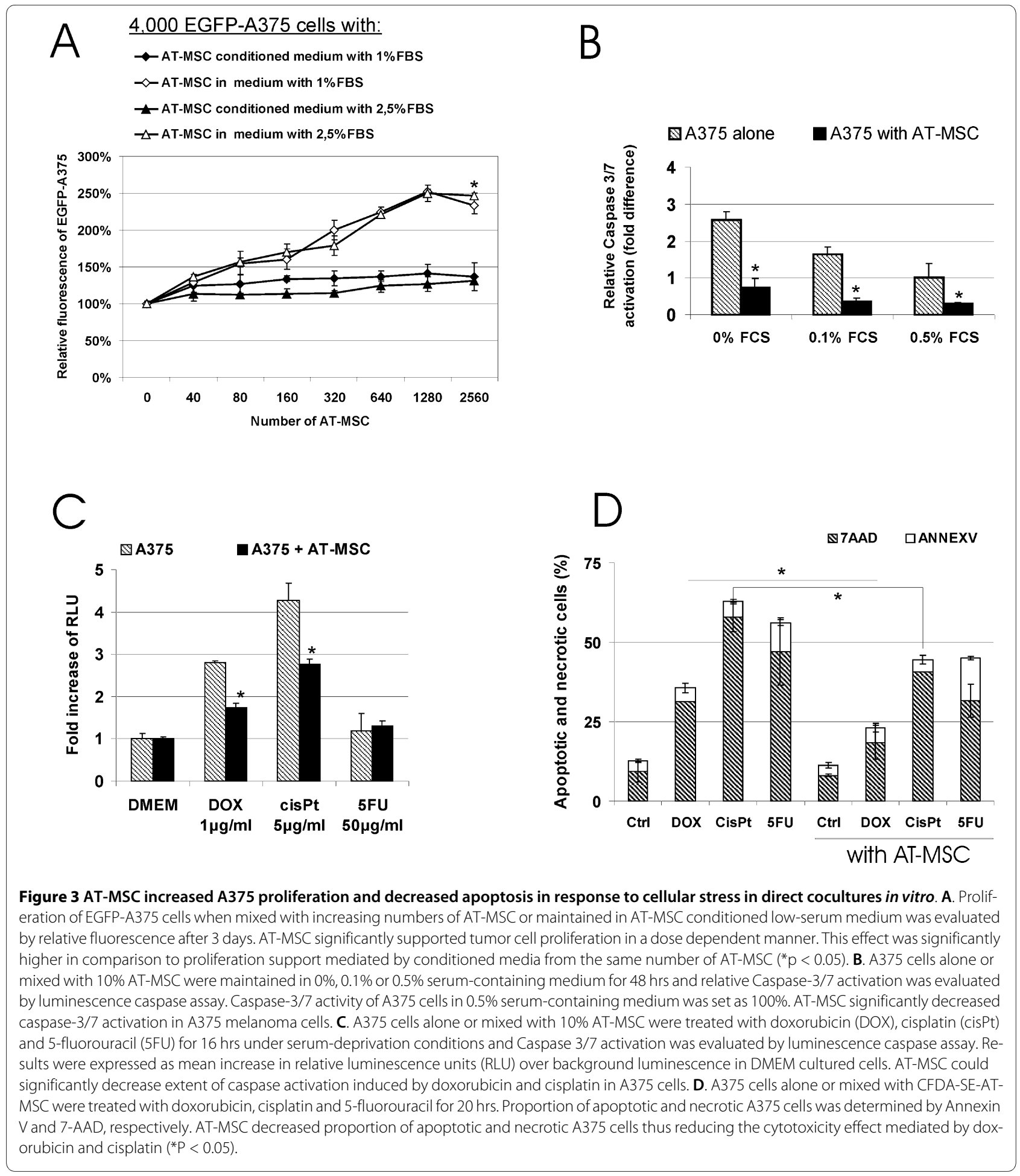

melanoma growth promotion in vivo. Next, in order to confirm relevance of the VEGF increased secretion in vivo we injected group of animals with mixtures of A375/ AT-MSC (2:1 ratio) and treated them with neutralizing antibody against human VEGF (antiVEGF, Avastin). This treatment decreased tumor incidence in comparison to antiVEGF untreated A375/AT-MSC group to some extent and also resulted in lower average tumor burden confirming the role of VEGF in the AT-MSC mediated tumor growth support (Fig. 5C). 


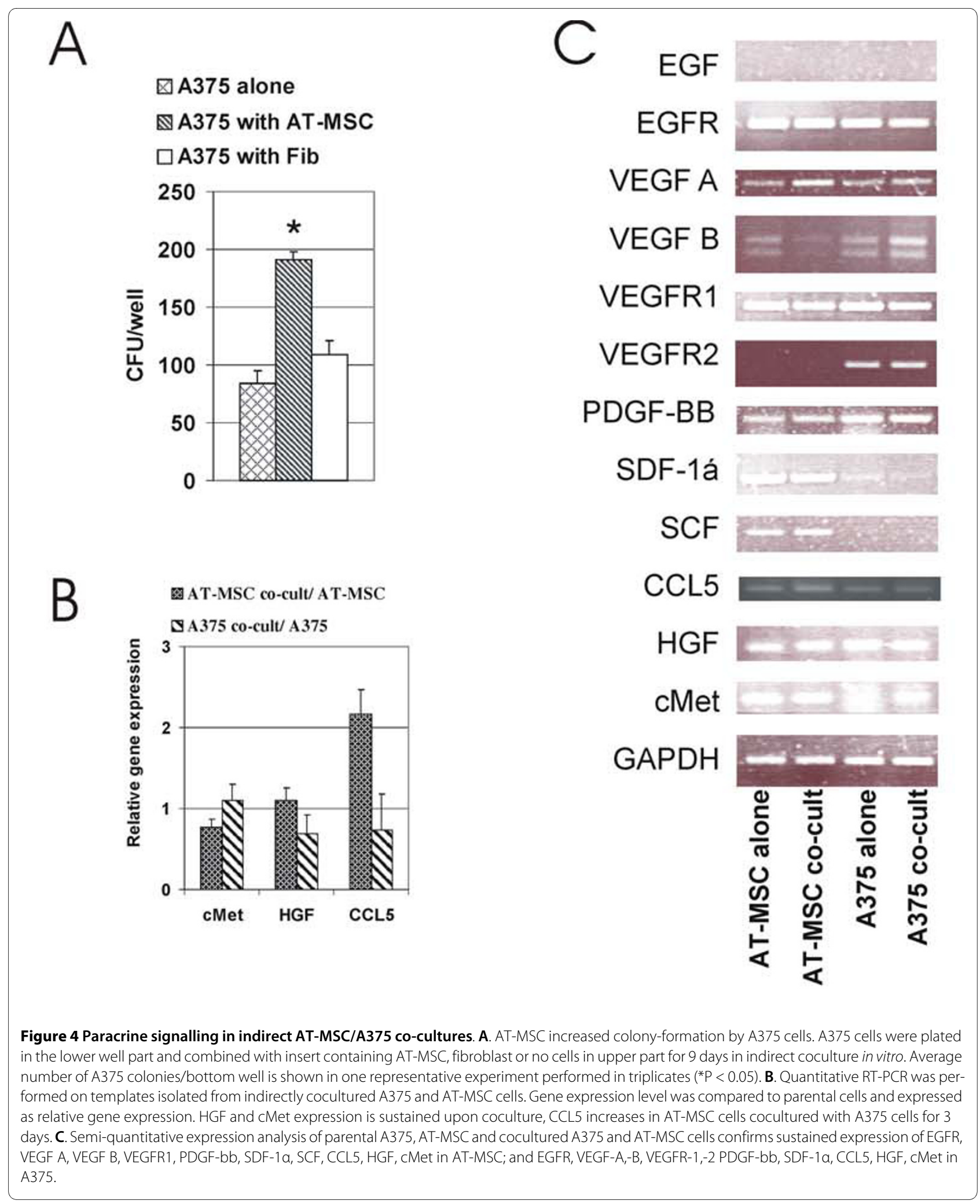




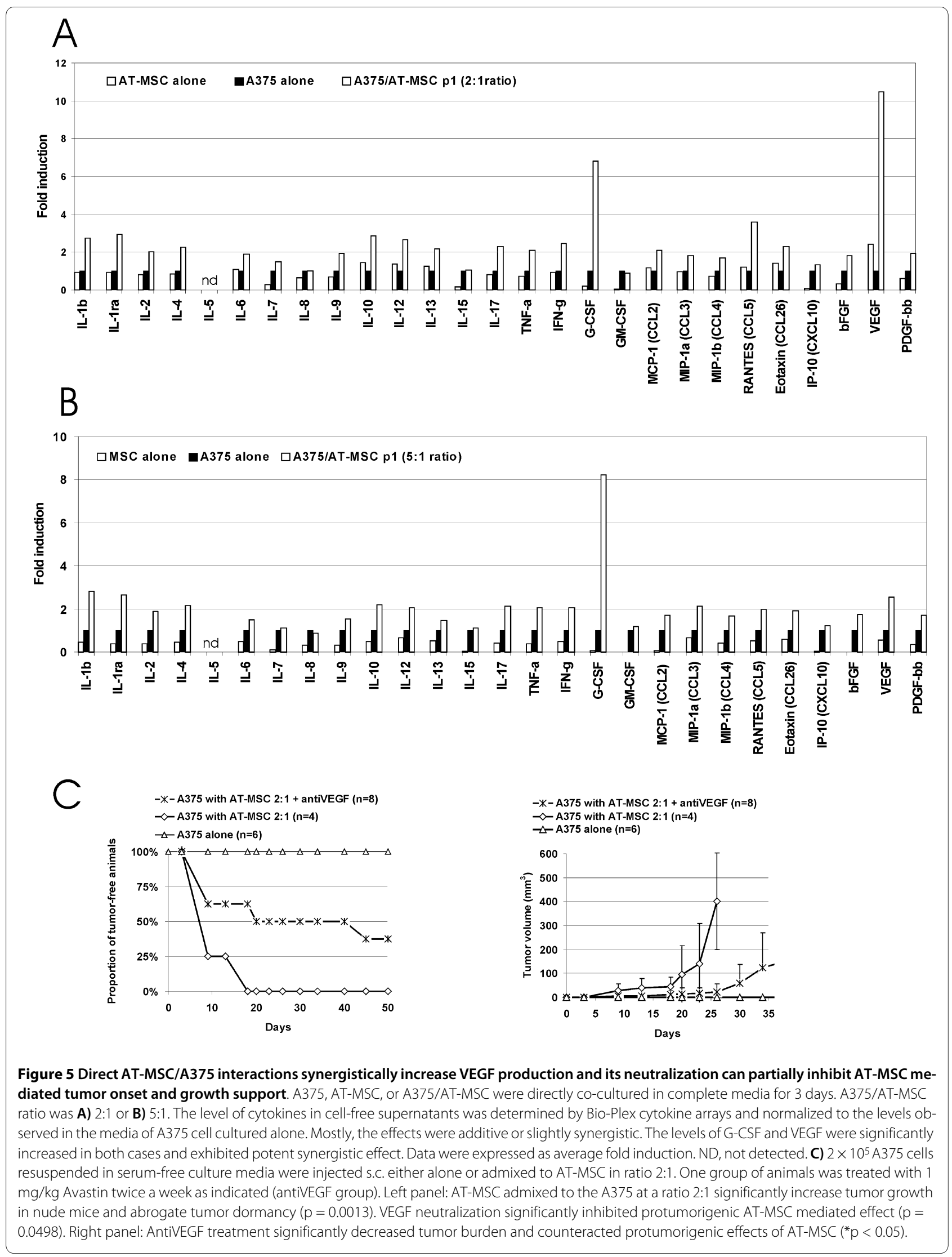




\section{Systemic administration of AT-MSC abrogates tumor dormancy and supports tumor growth via SDF-1 a/CXCR4 axis}

In order to evaluate whether AT-MSC-mediated tumor supporting effect is dependent on direct co-implantation and paracrine stimulation only, we decided to use different delivery route for AT-MSC - systemic intravenous administration frequently used in a clinical setting. ATMSC intravenous administration concomitant with the implantation of low A375 melanoma dose s.c. $\left(2 \times 10^{5}\right)$ lead to tumor growth in 7 out of 8 animals in contrast to no tumors growing without AT-MSC treatment $(0 / 4, \mathrm{p}=$ 0.00552 , data not shown). Even half of the melanoma cell dose was sufficient to mediate tumor growth in $67 \%$ of AT-MSC i.v. treated animals in contrast to $12.5 \%$ A375 alone s.c. inoculations (Fig. 6A). Consistently with previously published findings [17], we were not able to detect substantial proportion of EGFP expressing AT-MSC in subcutaneous A375 xenografts post-systemic administration at experiment endpoint by flow-cytometric analysis of single-cell suspension (data not shown). This might be caused by transient and/or early AT-MSC homing at the tumor site, detection limit of the system due to the outnumbering by rapidly proliferating tumor cells and/or limited AT-MSC proliferation within the tumor xenotransplant. However, we searched for the potential key mediator(s) that could have affected early homing/incorporation of AT-MSC into xenotrasplant implantation site. Role of SDF- $1 \alpha /$ CXCR 4 axis in this process was recognized [9-11]. We have confirmed sustained SDF-1 $\alpha$ production from AT-MSC $(1,756.5 \mathrm{pg} \pm 108.2 \mathrm{pg}$ per 50,000 AT-MSC). Moreover, $28.5 \%$ of A375 cells isolated from A375 xenograft expressed CXCR4 on cell surface (Fig. $6 \mathrm{~B})$. We hypothesized that even though the substantial amount of AT-MSC could not be detected in tumors, they actually might have homed very early into the site of tumor growth and SDF-1 $\alpha /$ CXCR4 axis could have been responsible for the homing. SDF- $1 \alpha$ signaling can be blocked by a CXCR4 antagonist AMD3100 - small molecule inhibitor, which enables to unravel role of this axis in the protumorigenic effects observed in vivo. In an attempt to abrogate tumor supportive effect of systemic AT-MSC administration on A375 xenograft, animals injected with A375s.c./AT-MSC i.v. were treated with $1.25 \mathrm{mg} / \mathrm{kg}$ AMD3100 every other day. Average tumor volume was decreased in AMD3100 treated group in comparison to control, but tumor incidences remained unaffected (Fig. 6C). These data indicated that SDF-1 $\alpha /$ CXCR4 axis contributed to AT-MSC mediated tumor growth support; however additional mechanism(s) might be responsible for tumor dormancy abrogation upon systemic injection of AT-MSC.

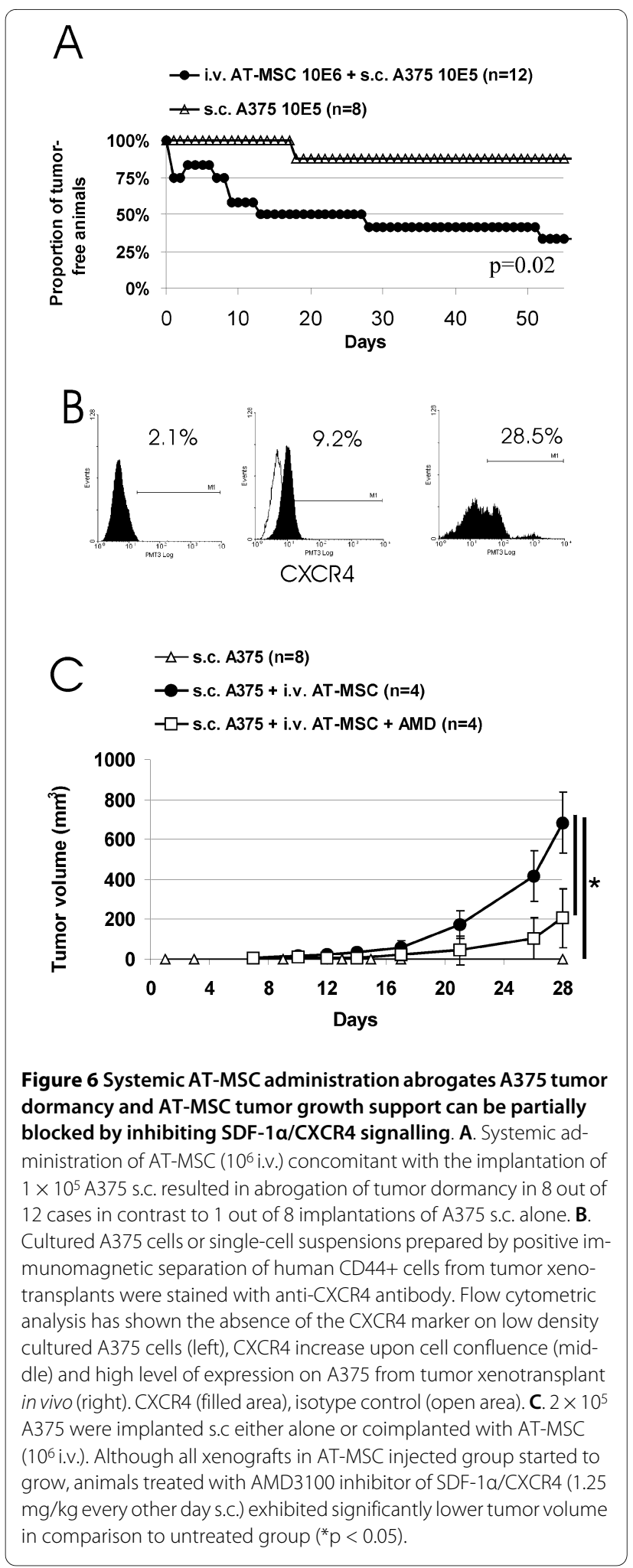




\section{AT-MSC did not promote growth of glioblastoma cells $8 \mathrm{MGBA}$ in vitro and in vivo}

In order to determine whether AT-MSC have tumor supportive effect on different tumor cell type, we have performed coculture experiments in vitro with glioblastoma cells 8MGBA. 8MGBA cells stably expressing EGFP were mixed with increasing amounts of AT-MSC or conditioned medium produced from corresponding amount of AT-MSC cells. Output fluorescence was proportional to the number of EGFP-8MGBA cells and was not influenced by the amount of admixed AT-MSC cells. Soluble factors did not support EGFP-8MGBA proliferation and directly cocultured EGFP-8MGBA/AT-MSC cocultures exhibited proliferation inhibition at highest 8MGBA/ATMSC proportions (Fig. 7A). Neither local coinjection nor systemic AT-MSC administration promoted glioblastoma xenograft growth in vivo. Implantation of high glioblastoma cell dose s.c. $\left(10^{7} 8 \mathrm{MGBA}\right)$ concomitantly with systemic AT-MSC administration resulted in $37.5 \%$ tumor incidence by day 55 (3 out of 8), which represented significant tumor growth suppression $(\mathrm{p}=0.0304)$. AT-MSC showed tendency to decrease tumor incidence upon admixing to low 8MGBA glioblastoma cells dose (Fig. 7B), even though expression analysis has shown similar expression pattern for the 8MGBA except for constitutive CXCR4 expression in comparison to melanoma cells A375 (Fig. 7C). Quantitative analysis has unravelled lower cMet receptor expression and significantly higher SDF$1 \alpha$ mRNA level in 8MGBA (Fig. 7C). Multiplex cytokine analysis was performed in order to quantitatively evaluate a paracrine signalling in tumor/AT-MSC cocultures. Combined coculture of 8MGBA/AT-MSC (2:1) exhibited increased secretion of IL- 6 , IFN- $\gamma$, G-CSF and additive effects for most of them. Overall outcome demonstrated several fold higher cytokine levels in 8MGBA/AT-MSC cocultures which might have been responsible for the observed inhibitory effect due to the synergistic action of these soluble factors (Fig. 7D).

Taken together, we have demonstrated both protumorigenic and antitumorigenic effect AT-MSC on malignant cell behaviour dependent on the mutual interplay between malignant and stromal cells to each other.

\section{Discussion}

MSC introduction into clinical studies has brought a lot of excitement about their beneficial effect in severe pathologic situations. Anyhow, patients treated with experimental therapies have to be aware of potential unknown effects. All determinants of MSC-mediated influence on tumor behaviour have not been fully characterized so far. Discrepancies amongst several studies reflect the complexity of tumor parenchyma/non-malignant stromal cells' interplay. Adherent multipotent progenitor cells produce plethora of cytokines (Fig. 5) [27-
29], that may modify tumor cell proliferation, metastasis, self-renewal, responses to cytotoxic stimuli, migration, and/or adhesion. Prosurvival action of MSC might be critical in the context of cellular stress as demonstrated in experiments performed under nutrients/cell limiting conditions in vitro (Fig. 3). Overall the outcome is clearly dependent on responsiveness of particular tumor cells in question and MSC-mediated changes upon interaction. Moreover, MSC physiological function implies their ability to function as niche marker cells possibly creating microregenerative niche for tumor cells that may facilitate to overcome hostile conditions in vivo [30,31]. Mutual tumor/MSC interplay leads to functional MSC changes even under tumor-cell produced soluble factors $[14,32]$. MSC do not seem to affect relative tumor growth rates in vivo, but substantially change tumor incidences for limiting numbers of inoculated cells [17]. Even soluble factors produced by AT-MSC were sufficient to enable the tumor (initiating) cells to overcome nutrition deprivation and engraft within the hostile microenvironment in vivo (Fig. 2A). Similar tumor promoting effects were reported for coinjection of fibroblasts or fibroblast conditioned medium with tumor epithelial cells supporting a role for soluble factors such as IL-6 [33-35]. These observations favour the hypothesis of MSC creating protective regenerative microenvironment by paracrine effects and/ or direct interaction.

Our observations that systemic AT-MSC administration could increase melanoma xenograft incidence in immunocompromised host might be also relevant for the future clinical trials. Dormant tumors frequently present in patients do not progress into growing tumors unless angiogenic switch occurs [36]. Whether the systemic MSC administration might present such stimulus in therapeutic approaches remains to be further carefully observed. MSC incorporation into tumors can be altered by anti-inflammatory treatment thereby abrogating inhibitory effect of MSC on pancreatic tumor growth [37]. Hung et al. have shown MSC incorporation into established subcutaneous HT-29 xenotransplants accompanied by loss of mesenchymal concomitant with endothelial marker expression [38]. Tumor-driven differentiation of MSC into phenotype of activated fibroblasts was described as another mechanism relevant for the MSC-mediated tumor progression [14,32]. However, Djouad et al. could not confirm MSC incorporation into the tumor on syngeneic model and they have supported the idea of systemic immunosuppression as a mechanism facilitating increased tumor incidence in syngeneic situation in vivo [17].

Direct coculture of AT-MSC with glioblastoma cells has shown the absence of proliferation support in vitro being in line with decreased tumor incidences in 8MGBA/AT-MSC inoculations (Fig. 7). Both tumor cell 


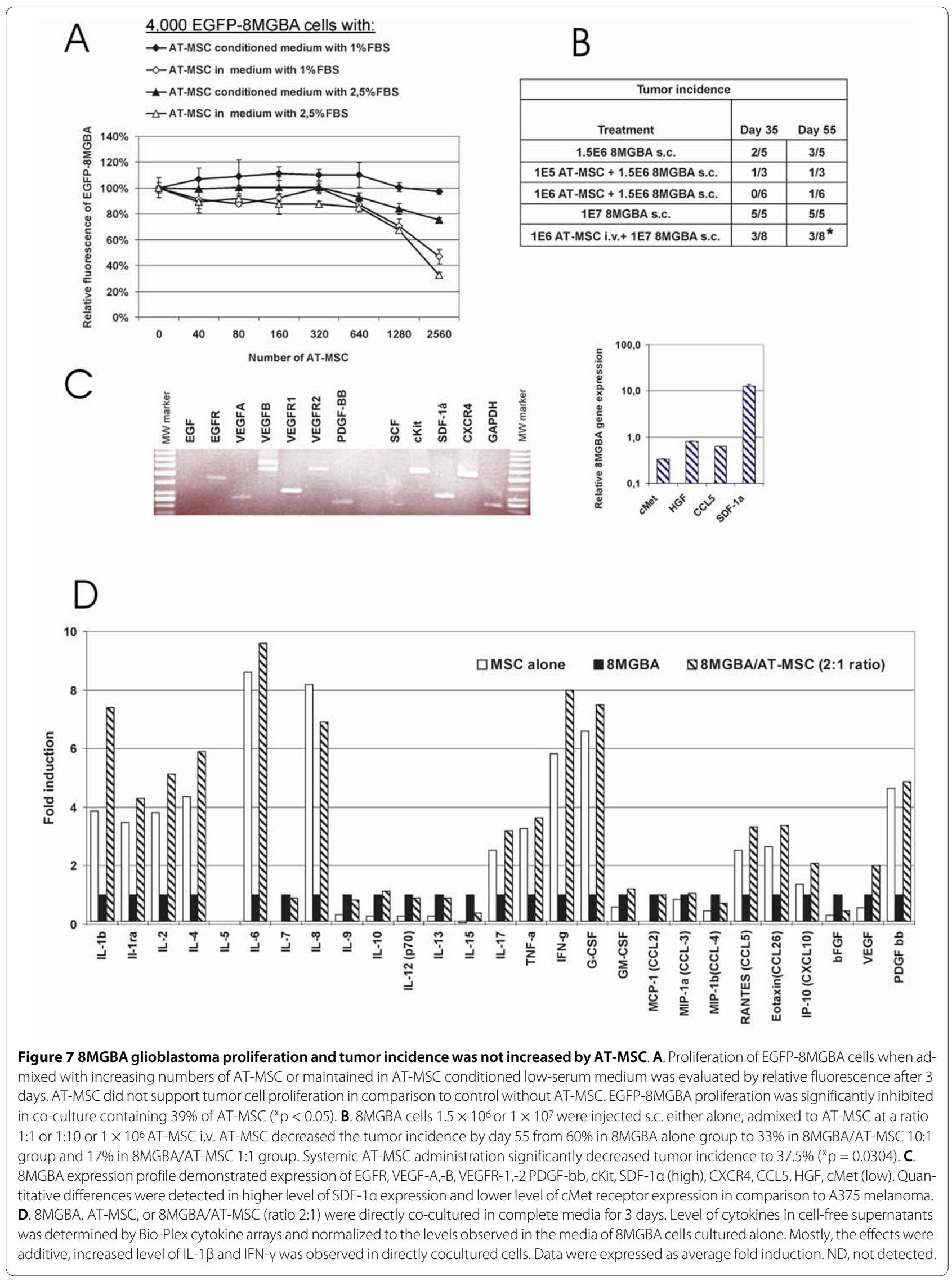


types express HGF, VEGF-A and PDGF-bb - growth factors implicated in the tumor-directed MSC migration (Fig. 4C and 7C) [27,39-41]. Moreover, expression of endothelial markers such as VEGFR1 and VEGFR2 on AT-MSC suggests the AT-MSC potential to contribute to tumor vasculature and/or premetastatic niche formation $[30,42]$. High level of G-CSF, renowned for the promotion of survival of leukocytes and their recruitment to the tissues, was detected in tumor/AT-MSC cocultures and contributed to formation of proinflammatory microenvironment [43]. Most prominent synergistic increase of VEGF in A375/AT-MSC cocultures indicated its potential role in tumor promotion. We suggest that high local VEGF concentrations contributed to the protumorigenic action of AT-MSC, although its neutralization could not completely abrogate AT-MSC effects indicative of role of other cytokines as well (Fig. 5C). According to the previously published data, CCL5 increase could contribute to higher metastatic potential of tumor cells rather than the proliferation increase [15]. Our experimental data confirm that both local and systemic AT-MSC administration exerted protumorigenic action on A375. Intravenous AT-MSC delivery resulted in abrogation of tumor dormancy (Fig. 6A). It has been previously proposed that SDF-1 $\alpha /$ CXCR4 signalling played role in AT-MSC homing within the sites of tumor formation and recently published data have demonstrated that inhibition of this axis by small molecule inhibitor AMD3100 could abrogate their migration towards prostate cancer cells [44]. More importantly, AMD3100 administration could abrogate glioblastoma regrowth by preventing post-irradiation recruitment of bone marrow progenitor cells [45]. Our data also demonstrate the contribution of SDF-1 $\alpha$ / CXCR4 signalling to AT-MSC-mediated A375 tumor growth. TGF $\beta$ is another key molecule playing a role in cell recruitment and affecting expression of other chemokines thereby also modulating tumor microenvironment [46]. TGF $\beta$ signalling might be another mechanism involved in tumor dormancy abrogation as SDF- $1 \alpha$ / CXCR4 inhibition was insufficient to counteract ATMSC mediated effect.

It seems as though AT-MSC produced tumor-inhibitory environment for given human glioblastoma cell line 8MGBA in our study similar to reported situations of cancer inhibiting inflammatory reaction [43]. We demonstrated that these glioblastoma cells were put into relatively cytokine "rich" environment produced by AT-MSC containing IL-1 $\beta$, IL-1ra, IL-2, IL-4, IL-6, IL-8, TNF- $\alpha$, IFN- $\gamma$, CCL5, CCL26, CXCL10, PDGF-bb. These could act in paracrine fashion alone or in combination to suppress glioblastoma cell growth such as described for synergistic inhibitory effect of TNF- $\alpha$ and IP-10 (CXCL10) [47]. Indeed, Nakamura et al. reported direct antiglioma role of unmanipulated MSC resulting in prolonged animal survival upon intracranial implantation [23].

Although tumor cells were put under the influence of similar AT-MSC produced proinflammatory and proangiogenic factors, we have observed contrastingly different responses. AT-MSC presented either tumor supportive or inhibitory effects depending on the inherent tumor cell properties and response to these factors.

\section{Conclusions}

Our study provided data to document both tumor-promoting and tumor-suppressive effects of AT-MSC on two different human tumor cell lines both in vitro and in vivo. Taken together, the complexity of tumor growth process and AT-MSC-mediated influence on tumor growth is reflected in this study. It highlights the necessity to study the tumor development in the context of tumor microenvironment to unravel determinants of tumor growth with the direct impact on therapeutic intervention [48]. Moreover, all these studies are inevitable to sufficiently describe potential MSC-attributable effects on the tumor behaviour in the light of wider MSC clinical use in both non-malignant and malignant therapeutic context.

\section{Methods}

\section{Cells and Chemicals}

Human melanoma cell lines A375 (ECACC No. 88113005), M4Beu, human fibroblasts (kindly provided by Dr. J. Bizik, CRI SAS, Bratislava), and glioblastoma multiforme 8MGBA (kindly provided by Dr. Perzelova, Med. School, Comenius University, Bratislava) were cultured in Dulbecco's modified Eagle medium (DMEM) supplemented with 5\% fetal calf serum (FCS) and Antibiotic-Antimycotic mix (GIBCO BRL, Gaithesburg, MD). EGFP-A375 and EGFP-8MGBA cells lines stably expressing enhanced green fluorescent protein were prepared as described elsewhere [49] and cultured as above. Cells were kept in humidified atmosphere and $5 \% \mathrm{CO}_{2}$ at $37^{\circ} \mathrm{C}$. AT-MSC were derived by plastic adherence technique as previously described in [24]. Briefly, AT-MSC cells were expanded from adherent cells obtained from stromalvesicular fraction upon collagenase type VIII digestion of lipoaspirate obtained from healthy persons, who provided an informed consent. Cells were expanded in low glucose $(1,000 \mathrm{mg} / \mathrm{ml})$ DMEM supplemented with $10 \%$ Mesenchymal stem cell stimulatory supplement (human, MSCSS) (StemCell Technologies, Grenoble, France) and Antibiotic-antimycotic (GIBCO BRL, Gaithesburg, MD). AT-MSC were characterized by surface marker expression as CD44+, CD73+, CD90+, CD105+, CD14-, CD34-, CD45- population and AT-MSC were capable of differentiation into adipocytes and osteoblasts. Conclusions were drawn from similar results of experiments performed with two different isolates if not specified otherwise. 
Cell-free AT-MSC conditioned medium was collected from $80 \%$ confluent AT-MSC cultures maintained in serum-free DMEM for $24 \mathrm{hrs}$ and used for inoculations in vivo.

All chemicals were purchased from Sigma (St. Louis, $\mathrm{MO})$ if not stated otherwise.

\section{Tumor cell and AT-MSC cocultures}

For proliferation evaluation, triplicates of 4,000 EGFPA375 or EGFP-8MGBA tumor cells/well were seeded in black-walled 96-well plates (Greiner Bio-One Intl. AG) with or without increasing numbers of AT-MSC for overnight. Same amounts of AT-MSC in triplicates were seeded in parallel 96-well plates in 1\% and 2.5\% FBS containing DMEM for preparation of AT-MSC conditioned media. This media after overnight incubation was transferred into corresponding wells to evaluate tumor cell proliferation in AT-MSC conditioned medium. Medium was replaced every day and relative proliferation was evaluated by PolarStar OPTIMA reader (BMG Labtechnologies, Offenburg, Germany) on day 3. Values were expressed as means of relative fluorescence $\pm \mathrm{SD}$, where EGFP tumor cell fluorescence in appropriate medium DMEM without AT-MSC was set to $100 \%$ by default. It was previously determined, that there was linear correlation between fluorescence intensity and number of EGFP expressing cells under these experimental culture conditions.

For apoptosis evaluation, quadruplicates of 15,000 tumor cells/well were seeded either alone or into wells containing 1,500 AT-MSC/well in 96-well plates for overnight. Cells were washed and treated with $0 \%, 0.1 \%$ and $0.5 \%$ serum containing media for 3 days to determine the extent of serum-deprivation induced apoptosis. Cells were treated with $1 \mu \mathrm{g} / \mathrm{ml}$ doxorubicin, $5 \mu \mathrm{g} / \mathrm{ml}$ cisplatin or $50 \mu \mathrm{g} / \mathrm{ml} 5$-fluorouracil in serum-free or $5 \%$ serum containing media for $16 \mathrm{hrs}$ to evaluate extent of cytotoxicity. Caspase-3/7 activation was determined by CaspaseGlo 3/7 Assay (Promega, Madison, WI) on LUMIstar GALAXY reader (BMG Labtechnologies, Offenburg, Germany). Values were expressed as fold increase in relative luminescence units (RLU) in comparison to tumor cells maintained in media alone.

For flow cytometric determination of apoptotic and necrotic cell proportions in direct cocultures, adherent AT-MSC were labelled with $5 \mu \mathrm{M}$ carboxy-fluorescein diacetate, succinimidyl ester (CFDA-SE, Molecular Probes, Eugene, OR) in a serum-free DMEM for $15 \mathrm{~min}$ at $37^{\circ} \mathrm{C}$. Medium was replaced for standard culture medium for overnight incubation. 100,000 tumor cells/ well were seeded with or without 10,000 CFDA-SE-AT$\mathrm{MSC} /$ well in duplicates in 24-well plates and serumdeprived for overnight. Cells were treated with $200 \mathrm{ng} / \mathrm{ml}$ doxorubicin, $5 \mu \mathrm{g} / \mathrm{ml}$ cisplatin or $50 \mu \mathrm{g} / \mathrm{ml} 5$-fluorouracil in $5 \%$ serum containing media for $20 \mathrm{hrs}$. Apoptotic cells were stained with Phycoerythrin-labelled Annexin V (Invitrogen, Carlsbad, CA); dead cells were detected with 7-AAD viability dye. Stained cells were analyzed using an EPICS ALTRA flow cytometer (Beckman Coulter, Fullerton, CA) equipped with Expo 32 program.

Colony-formation ability was evaluated in indirect tumor cell coculture with AT-MSC or fibroblasts. Tumor cells $\left(280 / \mathrm{cm}^{2}\right.$ in 6 -well plates) were plated into wells and combined with AT-MSC or fibroblasts physically separated in upper compartment and seeded on $0.4 \mu \mathrm{m}$ cell culture inserts $\left(5 \times 10^{4}\right.$ cell/insert) (Nalge Nunc International, Rochester, NY). Cells were maintained for 9 days in standard culture media. Average total number of A375 colonies per well was counted after Giemsa-Romanowski staining. Conclusions were drawn from three independent experiments.

\section{Analysis of gene expression}

Tumor cells were cultured with or without AT-MSC seeded on $0.4 \mu \mathrm{m}$ inserts $\left(10^{5}\right.$ cells/insert) (Nalge Nunc International, Rochester, NY) for 3 days. Total RNA was isolated from $0.5 \times 10^{6} 8 \mathrm{MGBA}, \mathrm{A} 375$, AT-MSC, A375 co-cultured cells and AT-MSC co-cultured cells collected from inserts by RNeasy mini kit (Qiagen, Hilden, Germany) and treated with RNase-free DNase (Qiagen, Hilden, Germany). RNA was reverse transcribed with RevertAid $^{\mathrm{TM}} \mathrm{H}$ minus First Strand cDNA Synthesis Kit (Fermentas, Hanover, MD). 200 ng of cDNA was subject to standard PCR performed in 1× PCR Master Mix (Fermentas, Hanover, MD) with 35 cycles and gel resolved on $2 \%$ agarose or $4 \%$ MetaPhor ${ }^{\circ}$ Agarose (Lonza, Rockland, ME, USA) for qualitative analysis.

Quantitative PCR was performed in $1 \times$ ABsolute $^{\mathrm{TM}}$ QPCR SYBR ${ }^{\circ}$ Green Mix (ABgene, Surrey, UK), $0.16 \mu \mathrm{M}$ primers and $500 \mathrm{ng}$ of template cDNA on RotorGene 2000 (Corbett Research, Sydney, Australia) and analyzed by RotorGene Software version 4.6. Primer sequences were used as previously published [15,27]. Relative gene expression change was calculated according to the formula Fold increase $=\left(\text { reaction efficiency }{ }^{*} 2\right)^{\Delta \Delta \mathrm{Ct}}$, where

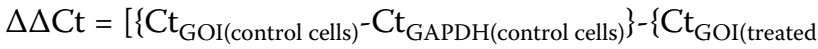
cells) $\left.\left.-\mathrm{Ct}_{\text {GAPDH(treated cells })}\right\}\right]$. GAPDH expression was taken as endogenous reference gene (GOI = gene of interest). Analysis was performed twice in triplicates and data expressed as means \pm SE.

\section{CXCR4 expression}

CXCR4 surface expression was analyzed in A375 cultures, or A375 cells isolated from tumor xenografts. Tumors were treated with $0.15 \%$ collagenase VIII, and $0.05 \mathrm{mg} / \mathrm{ml} \mathrm{DNAse} \mathrm{I} \mathrm{for} 45 \mathrm{~min}$ at $37^{\circ} \mathrm{C}$. Cells were sieved through $30 \mu \mathrm{m}$ pre-separation filters (Miltenyi Biotec, Bergisch Gladbach, Germany) to obtain single-cell sus- 
pension and immunomagnetically separated by EasySep human FITC selection kit (StemCell Technologies, Vancouver, BC, Canada) by positive selection with FITC conjugated human specific anti-CD44 antibody (Millipore, Billerica, USA). CD44 positive cells were stained with PEconjugated anti CXCR4 antibody (Invitrogen, Carlsbad, CA), anti-IgG2a isotype control (Invitrogen, Carlsbad, CA) and by flow cytometer. Representative result out of three independent experiments is shown.

\section{Cytokine secretion analysis}

50,000 A375 or 8MGBA, 25,000 AT-MSC cells alone plated in wells, 10,000 AT-MSC cells alone, 50,000 A375 (or 8 MGBA) cells mixed with AT-MSC (ratio $5: 1$ or $2: 1$ ) were cultured in complete culture medium for three days. Cell-free supernatants were collected and subjected to human Bio-Plex ${ }^{\mathrm{Tm}}$ 27-plex Cytokine Assay (Bio-Rad Laboratories Inc, Hercules, CA). Measurements were performed on Luminex 100 System (Luminex Corporation, Austin, TX) in duplicates with two different AT-MSC isolates. Results were expressed as means and relative cytokine expression was calculated by comparison to cytokine production from A375 (8MGBA) cells respectively.

SDF- $1 \alpha$ level was determined in cell free supernatants prepared as above by human SDF1- $\alpha$ Quantikine Immunoassay (R\&D Systems Inc.) on PolarStar OPTIMA reader (BMG Labtechnologies, Offenburg, Germany) as recommended by manufacturer.

\section{Experiments in vivo}

Six weeks old athymic nude mice (Balb/c-nu/nu) were used in accordance with institutional guidelines under the approved protocols. It was determined in preliminary studies that $10^{6} \mathrm{M} 4 \mathrm{Beu}$ cells, $1.5 \times 10^{6} \mathrm{~A} 375$ cells or $10^{7}$ 8 MGBA cells injected s.c. exhibit $100 \%$ tumor incidence. Following cell suspensions were injected in high dose coinjection studies: $1.5 \times 10^{6}$ A375 cells, $1.5 \times 10^{6} \mathrm{~A} 375+$ $1.5 \times 10^{5}$ AT-MSC (10\%AT-MSC), $1.5 \times 10^{6}$ A375 + $3 \times$ $10^{5}$ AT-MSC (20\% AT-MSC), $1 \times 10^{6}$ M4Beu cells, $1 \times 10^{6}$ M4Beu $+1 \times 10^{5}$ AT-MSC (10\% AT-MSC) (in $100 \mu \mathrm{l}$ of PBS s.c. into the flank). In an independent study animals received low tumor cell dose (tumor incidence 1/10) of 1 $\times 10^{4}, 1 \times 10^{5}$ or $2 \times 10^{5}$ A375 cells s.c as indicated. Groups of animals were directly coinjected with $1 \times 10^{6}, 1$ $\times 10^{5}, 1 \times 10^{4}$ AT-MSC in admixture s.c., or s.c administered $1 \times 10^{5}$ tumor cells were resuspended in AT-MSC conditioned media prior to injection. Independent group of animals was systemically administered with $1 \times 10^{6}$ AT-MSC i.v. into the lateral tail vein concomitantly with s.c. administration of A375 cells alone. Animals were subsequently treated with $1.25 \mathrm{mg} / \mathrm{kg}$ AMD3100 every other day s.c. or $1 \mathrm{mg} / \mathrm{kg}$ Avastin (Bevacizumab, Roche, kindly provided by National Cancer Institute, Bratislava) i.p. twice a week where indicated.

For glioblastoma xenograft study animals received low $\left(1.5 \times 10^{6}\right)$ 8MGBA cell dose cells s.c. Experimental groups of animals were directly coinjected with $1 \times 10^{6}$ or $1 \times 10^{5}$ AT-MSC in admixture s.c. Independent group of animals was systemically administered with $1 \times 10^{6} \mathrm{AT}$ MSC i.v. into the lateral tail vein concomitantly with s.c. administration of 8 MGBA high cell dose $\left(10^{7}\right)$.

Animals were regularly inspected for tumor incidence and designated tumor-free when no palpable rigid structures exceeding $1 \mathrm{~mm}$ in diameter could have been detected. Growing tumors were measured by calliper and volume was calculated according to formula volume = length $\times$ width $^{2} / 2$. Animals were sacrificed at the point, when the tumors exceeded $1 \mathrm{~cm}$ in diameter. Results were evaluated as mean volume $\pm \mathrm{SE}$.

\section{Statistical analysis}

Student's $\mathrm{t}$ test was used for comparison between the groups, differences in tumor incidences were evaluated by log-rank test, $\mathrm{P}$ value $<0.05$ was considered significant.

\section{List of abbreviations}

bFGF: basic fibroblast growth factor; cMet: hepatocyte growth factor receptor; CXCR4: SDF-1 $\alpha$ (CXCL12) receptor; EGF: epidermal growth factor; EGFR: epidermal growth factor receptor; FBS: foetal bovine serum; GAPDH: glyceraldehyde 3-phosphate dehydrogenase; GCSF: granulocyte-colony stimulating factor; GM-CSF: granulocyte monocyte-colony stimulating factor; HGF: hepatocyte growth factor; IFN-g: interferon $\gamma$; IL: interleukin; MCP-1 (CCL2): monocyte chemoattractant protein-1, chemokine CCL2; MIP-1a (CCL3): macrophage inflammatory protein-1alpha; MIP-1b (CCL4): macrophage inflammatory protein-1beta; PDGF-bb: plateletderived growth factor; RANTES (CCL5): Regulated on Activation, Normal T-cell Expressed and Secreted, chemokine CCL5; SCF: stem cell factor; SDF-1 $\alpha$ : stromaderived factor $1 \alpha$, chemokine CXCL12; TNF-a: tumor necrosis factor $\alpha$; VEGF: vascular endothelial growth factor; VEGFR: vascular endothelial growth factor receptor.

\section{Competing interests \\ The authors declare that they have no competing interests.}

\section{Authors' contributions}

LK designed study, performed experiments, wrote and drafted manuscript, MM performed flow cytometry analysis and EGFP cells, KH participated on in vivo experiments, VA isolated and established AT-MSC cultures, CA provided grant support, coordination and mentorship. All authors have read and approved the final manuscript.

\section{Acknowledgements}

We thank M. Dubrovcakova and V. Frivalska for excellent technical assistance, D. Guba, M.D., Institute of Medical Cosmetics, Bratislava, for providing material for 
AT-MSC isolation, Dr. L. Hunakova for help with flow cytometry analysis and Dr. D. Cholujova for technical help with Multiplex cytokine assay.

The study was supported by the VEGA grant No. 2/7060/27, APV grant No. APVV-0260-07, financial support was obtained from FIDURA Capital Consult $\mathrm{GmbH}$, Munich, SPP Foundation and grant support awarded by the League against Cancer.

\section{Author Details}

Laboratory of Molecular Oncology, Cancer Research Institute, Slovak Academy of Sciences, Bratislava, Slovakia

Received: 30 July 2009 Accepted: 28 May 2010

Published: 28 May 2010

\section{References}

1. Giordano A, Galderisi U, Marino IR: From the laboratory bench to the patient's bedside: an update on clinical trials with mesenchymal stem cells. J Cell Physiol 2007, 211:27-35.

2. Le Blanc K, Ringdén O: Immunomodulation by mesenchymal stem cells and clinical experience. J Intern Med 2007, 262:509-25.

3. Picinich SC, Mishra PJ, Mishra PJ, Glod J, Banerjee D: The therapeutic potential of mesenchymal stem cells. Cell- \& tissue-based therapy. Expert Opin Biol Ther 2007, 7:965-73.

4. Psaltis PJ, Zannettino AC, Worthley SG, Gronthos S: Concise review: mesenchymal stromal cells: potential for cardiovascular repair. Stem Cells 2008, 26:2201-10

5. Uccelli A, Moretta L, Pistoia V: Mesenchymal stem cells in health and disease. Nat Rev Immunol 2008, 8:726-36.

6. Ning $H$, Yang $F$, Jiang $M$, Hu L, Feng $K$, Zhang J, Yu Z, Li B, Xu C, Li Y, Wang J, Hu J, Lou X, Chen H: The correlation between cotransplantation of mesenchymal stem cells and higher recurrence rate in hematologic malignancy patients: outcome of a pilot clinical study. Leukemia 2008 22:593-9.

7. Valtieri M, Sorrentino AJ: The mesenchymal stromal cell contribution to homeostasis. Cell Physiol 2008, 217:296-300.

8. Li H, Fan X, Houghton J: Tumor microenvironment: the role of the tumor stroma in cancer. J Cell Biochem 2007, 101:805-15.

9. Joyce JA, Pollard JW: Microenvironmental regulation of metastasis. Nat Rev Cancer 2009, 9:239-52.

10. Hu M, Polyak K: Molecular characterisation of the tumor microenvironment in breast cancer. Eur J Cancer 2008, 44:2760-5.

11. Hall B, Andreeff M, Marini F: The participation of mesenchymal stem cells in tumor stroma formation and their application as targeted-gene delivery vehicles. Handb Exp Pharmacol 2007, 180:263-83.

12. Roorda BD, ter Elst A, Kamps WA, de Bont ES: Bone marrow-derived cells and tumor growth: contribution of bone marrow-derived cells to tumor micro-environments with special focus on mesenchymal stem cells. Crit Rev Oncol Hematol 2009, 69:187-98.

13. Stagg J: Mesenchymal stem cells in cancer. Stem Cell Rev 2008, 4:119-24.

14. Mishra PJ, Mishra PJ, Humeniuk R, Medina DJ, Alexe G, Mesirov JP, Ganesan S, Glod JW, Banerjee D: Carcinoma-associated fibroblast-like differentiation of human mesenchymal stem cells. Cancer Res 2008 68:4331-9.

15. Karnoub AE, Dash AB, Vo AP, Sullivan A, Brooks MW, Bell GW, Richardson AL, Polyak K, Tubo R, Weinberg RA: Mesenchymal stem cells within tumor stroma promote breast cancer metastasis. Nature 2007. 449:557-63.

16. Ramasamy R, Lam EW, Soeiro I, Tisato V, Bonnet D, Dazzi F: Mesenchymal stem cells inhibit proliferation and apoptosis of tumor cells: impact on in vivo tumor growth. Leukemia 2007, 21:304-10.

17. Djouad F, Bony C, Apparailly F, Louis-Plence P, Jorgensen C, Noël D: Earlier onset of syngeneic tumors in the presence of mesenchymal stem cells. Transplantation 2006, 82:1060-6.

18. Zhu W, Xu W, Jiang R, Qian H, Chen M, Hu J, Cao W, Han C, Chen Y: Mesenchymal stem cells derived from bone marrow favour tumor cell growth in vivo. Exp Mol Pathol 2006, 80:267-74.

19. Yu JM, Jun ES, Bae YC, Jung JS: Mesenchymal stem cells derived from human adipose tissues favour tumor cell growth in vivo. Stem Cells Dev 2008, 17:463-73.

20. Khakoo AY, Pati S, Anderson SA, Reid W, Elshal MF, Rovira II, Nguyen AT, Malide D, Combs CA, Hall G, Zhang J, Raffeld M, Rogers TB, Stetler-
Stevenson W, Frank JA, Reitz M, Finkel T: Human mesenchymal stem cells exert potent antitumorigenic effects in a model of Kaposi's sarcoma. $J$ Exp Med 2006, 203:1235-47.

21. Qiao L, Xu Z, Zhao T, Zhao Z, Shi M, Zhao RC, Ye L, Zhang X: Suppression of tumorigenesis by human mesenchymal stem cells in a hepatoma model. Cell Res 2008, 18:500-7.

22. Qiao L, Xu ZL, Zhao TJ, Ye LH, Zhang XD: Dkk-1 secreted by mesenchymal stem cells inhibits growth of breast cancer cells via depression of Wnt signalling. Cancer Lett 2008, 269:67-77.

23. Nakamura K, Ito Y, Kawano Y, Kurozumi K, Kobune M, Tsuda H, Bizen A, Honmou O, Niitsu Y, Hamada H: Antitumor effect of genetically engineered mesenchymal stem cells in a rat glioma model. Gene Ther 2004, 11:1155-64.

24. Kucerova L, Altanerova V, Matuskova M, Tyciakova S, Altaner C: Adipose tissue-derived human mesenchymal stem cells mediated prodrug cancer gene therapy. Cancer Res 2007, 67:6304-13.

25. Kucerova L, Matuskova M, Pastorakova A, Tyciakova S, Jakubikova J, Bohovic R, Altanerova V, Alatner C: Cytosine deaminase expressing human mesenchymal stem cells mediated tumor regression in melanoma bearing mice. J Gene Med 2008, 10:1071-82.

26. Studeny M, Marini FC, Champlin RE, Zompetta C, Fidler IJ, Andreeff M: Bone marrow-derived mesenchymal stem cells as vehicles for interferon-beta delivery into tumors. Cancer Res 2002, 62:3603-8

27. Kendall SE, Najbauer J, Johnston HF, Metz MZ, Li S, Bowers M, Garcia E, Kim SU, Barish ME, Aboody KS, Glackin CA: Neural stem cell targeting of glioma is dependent on phosphoinositide 3-kinase signalling. Stem Cells 2008, 26:1575-86.

28. Kögler G, Radke TF, Lefort A, Sensken S, Fischer J, Sorg RV, Wernet P: Cytokine production and hematopoiesis supporting activity of cord blood-derived unrestricted somatic stem cells. Exp Hematol 2005, 33:573-83.

29. Kilroy GE, Foster SJ, Wu X, Ruiz J, Sherwood S, Heifetz A, Ludlow JW, Stricker DM, Potiny S, Green P, Halvorsen YD, Cheatham B, Storms RW, Gimble JM: Cytokine profile of human adipose-derived stem cells: expression of angiogenic, hematopoietic, and pro-inflammatory factors. J Cell Physiol 2007, 212:702-9.

30. da Silva Meirelles $L$, Caplan Al, Nardi NB: In search of the in vivo identity of mesenchymal stem cells. Stem Cells 2008, 26:2287-99.

31. Ailles LE, Weissman IL: Cancer stem cells in solid tumors. Curr Opin Biotechnol 2007, 18:460-6.

32. Spaeth EL, Dembinski JL, Sasser AK, Watson K, Klopp A, Hall B, Andreeff M, Marini F: Mesenchymal stem cell transition to tumor-associated fibroblasts contributes to fibrovascular network expansion and tumor progression. PLOS ONE 2009, 4:e4992.

33. Camps JL, Chang SM, Hsu TC, Freeman MR, Hong SJ, Zhau HE, von Eschenbach AC, Chung LW: Fibroblast-mediated acceleration of human epithelial tumor growth in vivo. Proc Natl Acad Sci USA 1990, 87:75-9.

34. Noël A, De Pauw-Gillet MC, Purnell G, Nusgens B, Lapiere CM, Foidart JM: Enhancement of tumorigenicity of human breast adenocarcinoma cells in nude mice by matrigel and fibroblasts. Br J Cancer 1993, 68:909-15.

35. Studebaker AW, Storci G, Werbeck UL, Sansone P, Sasser AK, Tavolari S, Huang T, Chan MW, Marini FC, Rosol TJ, Bonafé M, Hall BM: Fibroblasts isolated from common sites of breast cancer metastasis enhance cancer cell growth rates and invasiveness in an interleukin-6dependent manner. Cancer Res 2008, 68:9087-95.

36. Naumov GN, Folkman J, Straume O: Tumor dormancy due to failure of angiogenesis: role of the microenvironment. Clin Exp Metastasis 2009, 26:51-60

37. Kidd S, Caldwell L, Dietrich M, Samudio I, Spaeth EL, Watson K, Shi Y, Abbruzzese J, Konopleva M, Andreeff M, Marini FC: Mesenchymal stromal cells alone or expressing interferon-beta suppress pancreatic tumors in vivo, an effect countered by anti-inflammatory treatment. Cytotherapy 2010 in press.

38. Hung SC, Deng WP, Yang WK, Liu RS, Lee CC, Su TC, Lin RJ, Yang DM Chang CW, Chen WH, Wei HJ, Gelovani JG: Mesenchymal stem cell targeting of microscopic tumors and tumor stroma development monitored by noninvasive in vivo positron emission tomography imaging. Clin Cancer Res 2005, 11:7749-56.

39. Nakamizo A, Marini F, Amano T, Khan A, Studeny M, Gumin J, Chen J, Hentschel S, Vecil G, Dembinski J, Andreeff M, Lang FF: Human bone 
marrow-derived mesenchymal stem cells in the treatment of gliomas. Cancer Res 2005, 65:3307-18.

40. Schichor C, Birnbaum T, Etminan N, Schnell O, Grau S, Miebach S, Aboody K, Padovan C, Straube A, Tonn JC, Goldbrunner R: Vascular endothelial growth factor A contributes to glioma-induced migration of human marrow stromal cells (hMSC). Exp Neurol 2006, 199:301-10.

41. Sun T, Sun BC, Ni CS, Zhao XL, Wang XH, Qie S, Zhang DF, Gu Q, Qi H, Zhao $\mathrm{N}$ : Pilot study on the interaction between B16 melanoma cell-line and bone-marrow derived mesenchymal stem cells. Cancer Lett 2008, 263:35-43.

42. Kaplan RN, Riba RD, Zacharoulis S, Bramley AH, Vincent L, Costa C, MacDonald DD, Jin DK, Shido K, Kerns SA, Zhu Z, Hicklin D, Wu Y, Port JL, Altorki N, Port ER, Ruggero D, Shmelkov SV, Jensen KK, Rafii S, Lyden D: VEGFR1-positive haematopoietic bone marrow progenitors initiate the pre-metastatic niche. Nature 2005, 438:820-7.

43. Mantovani A, Allavena P, Sica A, Balkwill F: Cancer-related inflammation. Nature 2008, 454:436-44.

44. Lin G, Yang R, Banie L, Wang G, Ning H, Li LC, Lue TF, Lin CS: Effects of transplantation of adipose tissue-derived stem cells on prostate tumor. Prostate 2010 in press.

45. Kioi M, Vogel H, Schultz G, Hoffman RM, Harsh GR, Brown JM: Inhibition of vasculogenesis, but not angiogenesis, prevents the recurrence of glioblastoma after irradiation in mice. J Clin Invest 2010, 120:694-705.

46. Lazennec G, Richmond A: Chemokines and chemokine receptors: new insights into cancer-related inflammation. Trends Mol Med 2010, 16:133-44.

47. Enderlin M, Kleinmann EV, Struyf S, Buracchi C, Vecchi A, Kinscherf R, Kiessling F, Paschek S, Sozzani S, Rommelaere J, Cornelis JJ, Van Damme J, Dinsart C: TNF-alpha and the IFN-gamma-inducible protein 10 (IP-10/ CXCL-10) delivered by parvoviral vectors act in synergy to induce antitumor effects in mouse glioblastoma. Cancer Gene Ther 2009, 16:149-60.

48. Lazennec G, Jorgensen C: Concise review: adult multipotent stromal cells and cancer: risk or benefit? Stem Cells 2008, 26:1387-94

49. Matuskova M, Hlubinova K, Pastorakova A, Hunakova L, Altanerova V Altaner C, Kucerova L: HSV-tk expressing mesenchymal stem cells exert bystander effect on human glioblastoma cells. Cancer Lett 2010, 290:58-67.

doi: $10.1186 / 1476-4598-9-129$

Cite this article as: Kucerova et al., Tumor cell behaviour modulation by mesenchymal stromal cells Molecular Cancer 2010, 9:129

\section{Submit your next manuscript to BioMed Central} and take full advantage of:

- Convenient online submission

- Thorough peer review

- No space constraints or color figure charges

- Immediate publication on acceptance

- Inclusion in PubMed, CAS, Scopus and Google Scholar

- Research which is freely available for redistribution

Submit your manuscript at www.biomedcentral.com/submit
Ciomed Central 